the 12 item VCDQ; for chronic cough the 19-item LCQ), and patient satisfaction questionnaires and flexible laryngoscopy performed post therapy.

Results Eleven people have completed SLT over Skype ${ }^{\mathrm{TM}}$ to date, and all demonstrated improvement in symptoms following therapy. Patients with VCD showed a decrease in score on the VCDQ from median (range) $48(12-53)$ pre therapy to 40 (742) post therapy [minimal clinical important difference (MCID) 5]. Patients with chronic cough showed an increase on the LCQ from median (range) $6.4(4.6-8.2)$ pre therapy to 12.2 (1014.6) post therapy (MCID 1.3). Improvements in laryngeal tension and sensitivity were noted in all cases. All patients gave positive feedback in their patient satisfaction questionnaire scoring "very satisfied" or greater. On three occasions Skype connection problems delayed sessions by a few days.

Conclusions Virtual consultations provide the opportunity to treat patients in a more time efficient and practical way, and improvements in patient-reported symptoms and laryngeal appearances were similar to those of patients attending therapy sessions in chest clinic. This data gives support to pursue formalised tariffs for a specialised telehealth service. We feel that Skype should continue as a regular therapy option for patients and other members of the multi-disciplinary team (MDT) should consider this method of therapy delivery.

\section{P113 THE USE OF LOCAL ANAESTHESIA IN IMPROVING THE PATIENT EXPERIENCE OF ARTERIAL BLOOD GASES: STUDENTS AND TRAINERS ARE STILL NOT GETTING THE MESSAGE}

'Z Mangera, ${ }^{1} \mathrm{C}$ Gunasekera, '1 Kinley, ${ }^{2} \mathrm{~J}$ King, ${ }^{1} \mathrm{~B}$ Walker, ${ }^{3} \mathrm{O}$ Cohen, ${ }^{4} \mathrm{P}$ Dilworth. ${ }^{1}$ University College London Medical School, London, UK; ${ }^{2}$ The Whittington Hospital, London, UK; ${ }^{3}$ University College Hospital, London, UK; ${ }^{4}$ Royal Free Hospital, London, UK

\subsection{6/thoraxjnl-2014-206260.254}

Background BTS guidelines (2008) states that "local anaesthesia (LA) should be used for all arterial blood gas (ABG) specimens except in emergencies ${ }^{1}$ as it improves the patient experience. A survey conducted in 2012 revealed only 5\% of junior doctors regularly use LA with ABGs. ${ }^{2}$ We were interested to determine if this has improved and establish whether medical students are being orientated to this practice.

Methodology This is a multicentre prospective study. A questionnaire survey was distributed to 4th year medical students and junior doctors affiliated with UCL Medical School. Questions related to their actual experiences of using LA with ABGs and barriers to using LA.

Results 94 medical students completed the questionnaire. Students used LA $17 \%$ of the time out of 54 supervised procedures. 29\% were actively discouraged from using LA by their supervising doctor. $10 \%$ felt the general culture amongst supervising doctors was resistant to using LA.

Amongst the 86 surveyed doctors, 91\% never or rarely $(<10 \%$ of the time) used LA, $5 \%$ sometimes ( $<25 \%$ of the time) and $3 \%$ used it regularly ( $>75 \%$ of the time). $65 \%$ of doctors were not aware that LA was advised in national guidance. $40 \%$ of respondents felt it would not reduce the pain of the procedure and 38\% did not know the technique involved of using LA.

Conclusion The use of LA is extremely poor as has been found previously. ${ }^{1}$ The reasons reflect a lack of awareness and a culture that is experienced from the moment the enter the clinical environment as medical students. In order to improve the patient experience we have introduced an intervention at the level of the medical school and junior doctor teaching which includes mandatory training and encouraging affiliated trusts to help create a culture where giving LA is the norm.

We will fully report on the results and success of our interventions.

\section{REFERENCES}

1 O'Driscoll, Howard LS, Davison AG. BTS guideline for emergency oxygen use in adult patients. Thorax. 2008; 63 (Suppl VI):vi1-vi73

2 Khan F et al. The Use of Local Anaesthesia For Arterial Blood Gas Sampling - A Multicentre Survey. Thorax 2012;67:A167-A168

\section{P114 WHAT SKILLS, EXPERIENCE AND TRAINING ARE NEED TO WORK IN INTEGRATED RESPIRATORY SPECIALIST ROLES AND HOW CAN WE ROLL THESE POSTS OUT IN THE UK?}

${ }^{1} \mathrm{NJ}$ Roberts, ${ }^{2} \mathrm{M}$ Ward, ${ }^{3} \mathrm{SS}$ Patel, ${ }^{4} \mathrm{~J}$ Yorke, ${ }^{5} \mathrm{~J}$ Williams, ${ }^{6} \mathrm{R}$ Walters, ${ }^{7} \mathrm{M}$ McKevitt, ${ }^{8} \mathrm{~S}$ Edwards. ${ }^{1}$ Glasgow Caledonian University, Glasgow, UK; ${ }^{2}$ Sherwood Forest Hospitals, Sutton in Ashfield, UK; ${ }^{3}$ Kings College Hospital London, London, UK; ${ }^{4}$ University of Manchester, Manchester, UK; ${ }^{5}$ Halton General Hospital, Runcorn, UK; ${ }^{6}$ Mansfield Community Hospital, Mansfield, UK; ${ }^{7}$ British Lung Foundation, London, UK; ${ }^{8}$ British Thoracic Society, London, UK

\subsection{6/thoraxjnl-2014-206260.255}

Aims There is an increased drive towards healthcare integration in the UK (UK) to adapt to new health care needs. In response to this, new ways of delivering care have been developed such as the provision of integrated respiratory specialists. This project set out to describe these roles, the key skills needed and how junior staff can aspire to these roles.

Methods Semi-structured telephone interviews with 12 integrated care specialists or those currently working with integrated respiratory care teams were undertaken to explore and discuss the role of integrated respiratory specialists.

Results Nine integrated specialists were interviewed (6 physicians, 2 nurses, 1 physiotherapist). One Specialist Trainee (StR), a general practitioner (GP) and a pharmacist were also interviewed. The integrated role was variable for participants; most were involved in MDTs, education for staff and developing guidance, with some involved in community or virtual clinics. Physicians were more likely to be involved in Acute Medical Unit reviews. Five interviewees had two joint leads with either two physicians, or a physician and a nurse.

Key skills identified by the interviewees included respiratory specialist knowledge, prior primary care experience as well as knowledge of the NHS, commissioning and social services processes. Specific training included conflict management, advanced communication and research and evaluation skills (health economics and service evaluation). Three areas were highlighted to support the establishment of new posts: clear details about the posts and standards of care; embedding integrated care into training; increasing visibility of this new area and gaining support from appropriate organisations.

Conclusions Interviewees highlighted that existing posts should be used as exemplars to provide junior staff with more information about these roles. Greater collaboration with professional societies such as the BTS, ERS and PCRS for education as well as to promote and advance these new potential career pathways should be undertaken.

Funded by the British Thoracic Society. 